\title{
Intramuscular hemangioma with extensive ossification: Unique radiographic and histologic features
}

\author{
Behdokht Nowroozizadeh, Di Lu* \\ Departments of Pathology, UC Irvine Medical Center, UC Irvine School of Medicine, Orange, USA
}

Received: August 22, 2016

Accepted: November 6, $2016 \quad$ Online Published: December 19, 2016

DOI: $10.5430 /$ crep.v4n1p59

URL: http://dx.doi.org/10.5430/crcp.v4n1p59

\begin{abstract}
Intramuscular hemangioma is a benign tumor representing $0.8 \%$ of all hemangiomas. Rarely intramuscular hemangioma presents with massive ossification in the mass that can be misleading and cause misdiagnoses, and unnecessary workups. We describe a case of intramuscular hemangioma with massive ossification in the left thigh of a 54-year-old man presented with pressure symptoms while exercising.
\end{abstract}

Key Words: Muscle lesion, Hemangioma, Ossification, Intramuscular hemangioma, Calcification

\section{INTRODUCTION}

Although hemangioma is a common benign tumor, particularly for cutaneous type of childhood, intramuscular hemangiomas are rare and comprise just $0.8 \%^{[1]}$ and $4.4 \%^{[2]}$ respectively for all hemangiomas. The typical features may include calcification among others. Ossification, especially when occurs in intra-muscular hemangioma of skeletal muscle, is rare ${ }^{[3,4]}$ In this report we describe a case of skeletal muscle hemangioma of thigh that contained abundant mature cortical bone formation.

\section{CASE REPORT}

A 54-year-old man came to our institution for evaluation of a large left thigh mass. He noticed the left thigh mass as early as he was only 7-year-old. The mass continuously grew in size. He denies any trauma, weakness or pain, but he complains of pressure symptoms while exercising.

The mass was palpable large, non-tender, measuring $15 \mathrm{~cm}$ in greatest dimension and locates in the lateral aspect of his left thigh. The mass is not adherent to overlying skin.
Motion of the right knee, hip, and ankle joints was normal. Skin has no abnormal changes. Neurovascular examination was within normal limits. Edema, varicose veins or inguinal lymphadenopathy were not identified.

Magnetic resonance imaging demonstrated an approximately $14.6 \mathrm{~cm} \times 6.5 \mathrm{~cm} \times 5.2 \mathrm{~cm}$ mass containing a tangle of flow voids. The mass has a $0.6 \mathrm{~cm}$ focus of internal hemorrhage and a $2.8 \mathrm{~cm}$ rounded structures. There also appeared to be a well-defined fat plane between the mass/vastus medialis and adjacent muscle and neurovascular structures of the anterior and medial compartments.

Angiographic images demonstrated rapid, florid arterial enhancement, contrast pudding, and tangled arteries. There were feeding vessels from the left profunda femoris and mild-to-distal superficial femoral artery. An enlarged early, draining vein drains into the superficial femoral vein proximally with additional smaller draining veins throughout the course of the superficial femoral vein (see Figure 1).

*Correspondence: Di Lu, M.D; Email: lud5@uci.edu; Address: 101 The City Dr S, Orange, CA 92868. Pathology department, USA. 


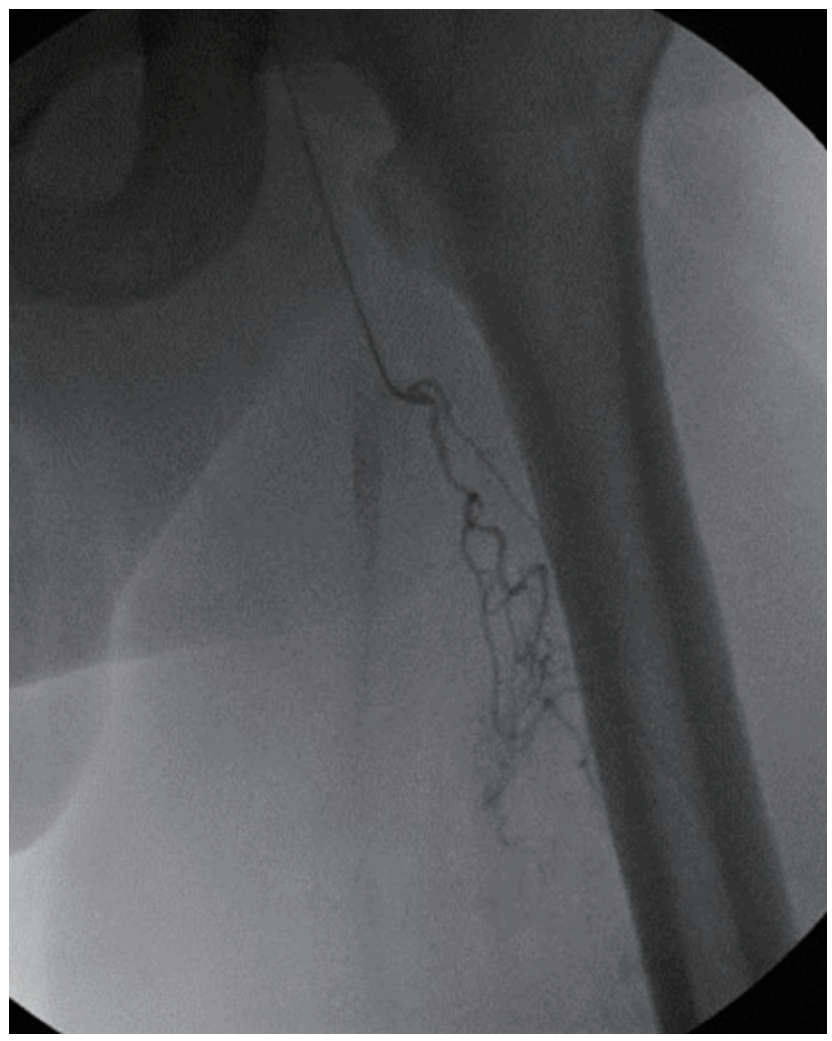

Figure 1. Angiographic image shows rapid, florid arterial enhancement, and tangled arteries. There were feeding vessels from the left profunda femoris and mild-to-distal superficial femoral artery.

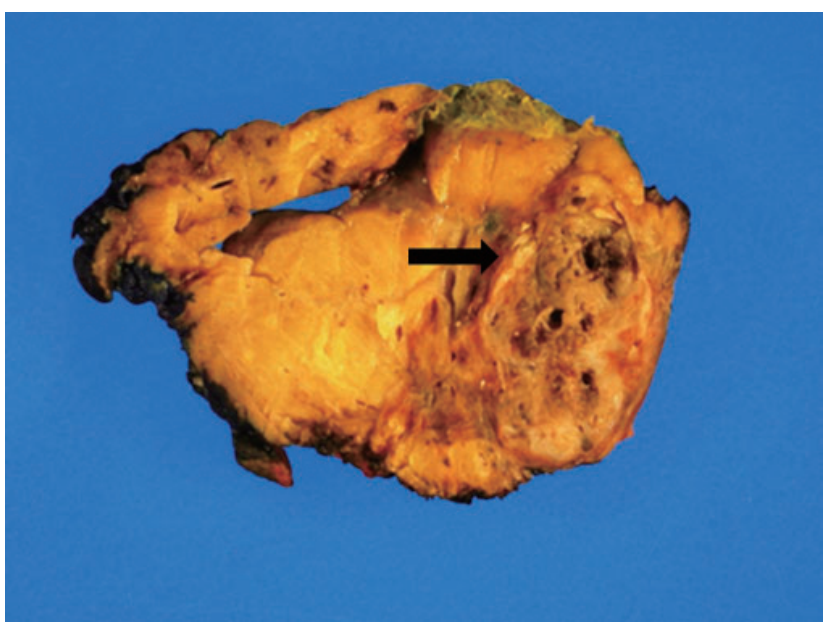

Figure 2. Gross examination demonstrated ill-defined red brown vascular tumor with circular calcification and extensive ossification (arrow)

He underwent wide local excision of his left thigh mass. Gross examination of the specimen demonstrated ill-defined red brown vascular tumor surrounded by skeletal muscle. The tumor measures $7.5 \mathrm{~cm} \times 7 \mathrm{~cm} \times 3 \mathrm{~cm}$ and weighs $98 \mathrm{~g}$. Cut section shows circular calcification and extensive ossification measuring $2.5 \mathrm{~cm} \times 2 \mathrm{~cm} \times 2 \mathrm{~cm}$ in size (see Figure 2).

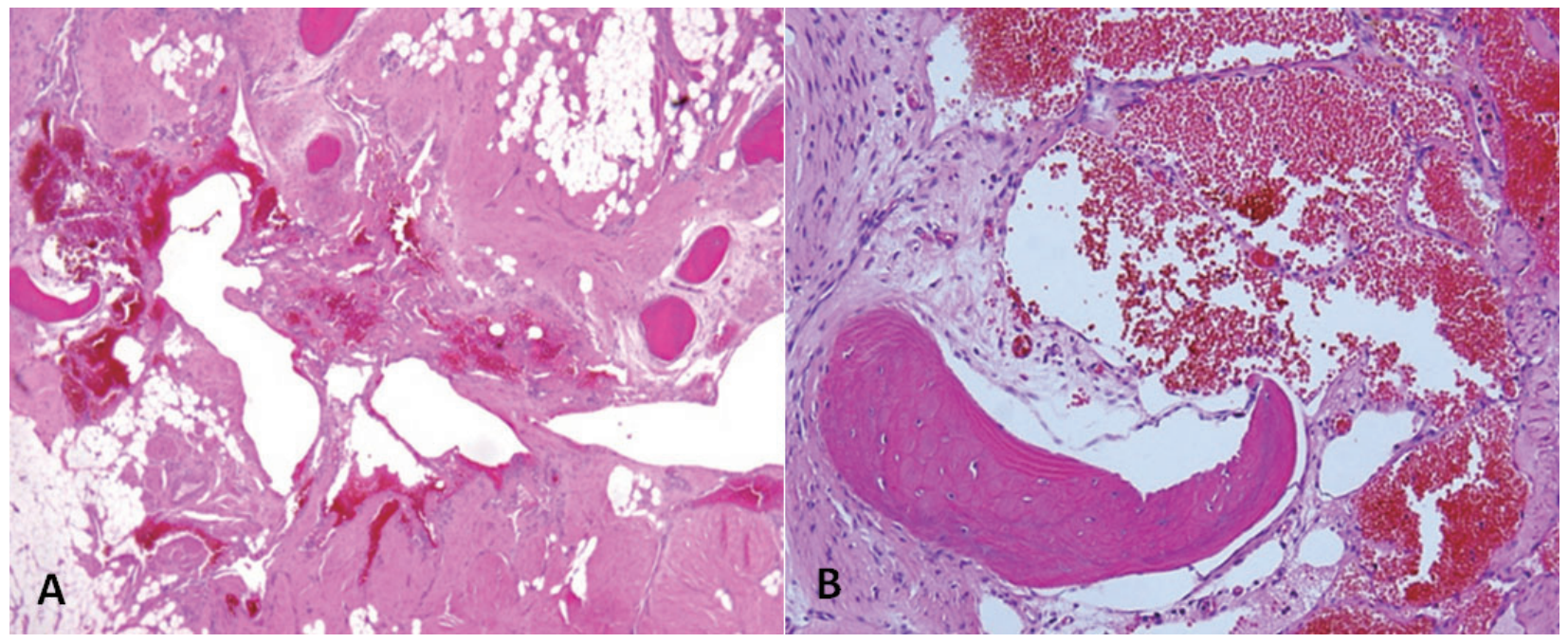

Figure 3. Photomicrograph of the lesion reveals variable sized and dilated blood vessels. These vessels are intermingled and embedded with trabeculae of lamellar bone in a fibrous stroma (A). Higher magnification showing dilated blood vessels are lined by benign flat endothelial cells. No bone marrow is seen (B).

Histological examination revealed a tumor with lobular configuration surrounded by skeletal muscle. The tumor consists of variable sized and dilated blood vessels which are lined by benign flat endothelial cells. These vessels are intermingled and embedded with trabeculae of lamellar bone in a fibrous stroma (see Figure 3). The findings are diagnostic of ossified intramuscular hemangioma. 


\section{DISCUSSION}

Common locations of hemangiomas are upper and lower extremities, the trunk, and the head and neck region. ${ }^{[4]}$ Intramuscular hemangiomas are rare (less than $1 \%$ of all hemangiomas). Usually, they present with enlarging painful mass, especially during exercise. Skin is usually unaffected. In some cases, dystrophic calcification can be formed due to long-standing organizing thrombi. However, ossification in hemangiomas is a very rare phenomenon. ${ }^{[4]}$ Histologic examination of this case reveals classic cavernous hemangioma components with area of mature bone formation surrounded by skeletal muscle. The differential diagnosis of the gross examination of ossified intramuscular hemangioma includes myositis ossificans, ossifying fibromyxoid tumor of soft parts, fibrodysplasia ossificans, synovial sarcoma, extraskeletal osteosarcoma, malignant giant cell tumor, and malignant schwannoma. ${ }^{[1,4]}$ However, only in ossified intramuscular hemangioma we can see hemangioma microscopic features like proliferation of the vascular channels that filled with red blood cells. In the other differential diagnoses we don't have hemangioma features; therefore, microscopic examinations and immunohistochemistry stains sort out most of these differentials and render a definite diagnosis.

\section{Conclunions}

Ossified intramuscular hemangioma should be considered for any enlarging intramuscular mass with ossification. Although hemangioma is a very common neoplasm, intramuscular hemangioma is rare and ossified intramuscular hemangioma is even rarer.

\section{CONFlicts OF InTEREST Disclosure}

The authors declare no conflicts of interest.

\section{REFERENCES}

[1] Chang PY, Lan HHC, Chen CCC, et al. Ossified Intramuscular Hemangioma. J Radiol Sci. 2012; 37: 39-43.

[2] Jiang TT, Cisa J, Desai P, et al. Intramuscular ossified hemangioma. Skeletal Radiol. 1995; 24: 538-40. PMid: 8545654. https : //doi.org/10.1007/BF00202155

[3] Barry LE, Louis AG, Michael K. Ossified skeletal muscle heman- gioma: radiologic and pathologic features. Skeletal Radiol. 1980; 5(1): 35-40. PMid: 6767278. https ://doi.org/10.1007/BF00 347096

[4] Keiko N, Tetsuji Y, Takashi M, et al. Ossified intramuscular hemangioma: Multimodality imaging findings. Journal of Clinical Imaging. 2001; 25: 368-72. https ://doi .org/10.1016/S0899-7071 (01 )00319-9 